\title{
Three-dimensional computed tomography image overlay facilitates thoracoscopic trocar placement
}

Hiroyuki Oizumi, MD, PhD, Hirohisa Kato, MD, PhD, Hikaru Watarai, MD, and Mitsuaki Sadahiro, MD, PhD, Yamagata, Japan

Although thoracoscopic surgical procedures have brought enormous benefits to the treatment of a variety of lesions, trocar placement can sometimes be problematic or even inadequate. Multidetector computed tomography (CT) has

From the Second Department of Surgery, Faculty of Medicine, Yamagata University, Yamagata, Japan.

Disclosures: Authors have nothing to disclose with regard to commercial support. Received for publication Aug 4, 2012; revisions received Feb 9, 2013; accepted for publication April 18, 2013; available ahead of print June 13, 2013

Address for reprints: Hiroyuki Oizumi, MD, PhD, Second Department of Surgery, Yamagata University, 2-2-2 Iida-Nishi, Yamagata 990-9585, Japan (E-mail: hohizumi@med.id.yamagata-u.ac.jp).

J Thorac Cardiovasc Surg 2013;146:720-1

$0022-5223 / \$ 36.00$

Copyright $(\odot 2013$ by The American Association for Thoracic Surgery

http://dx.doi.org/10.1016/j.jtcvs.2013.04.031 uncovered new possibilities in the planning of therapeutic strategies. Herein, we report the use of 3-dimensional (3D) images projected directly onto the patient's body surface to help surgeons identify the position of anatomic structures, thereby facilitating the optimum placement of thoracic ports.

\section{SIMULATION BY 3D-CT}

Images were obtained using 64-channel multidetector $\mathrm{CT}$, and $3 \mathrm{D}$ volume rendering was used to construct a solid image from $1.0-\mathrm{mm}$ data slices. The 3D images were processed by an AquariusNet (TeraRecon, San Mateo, Calif) or OsiriX (http://www.osirix-viewer.com) device, and image reconstruction was performed in real time in the operating room. The processing required no more than 7 minutes. ${ }^{1}$
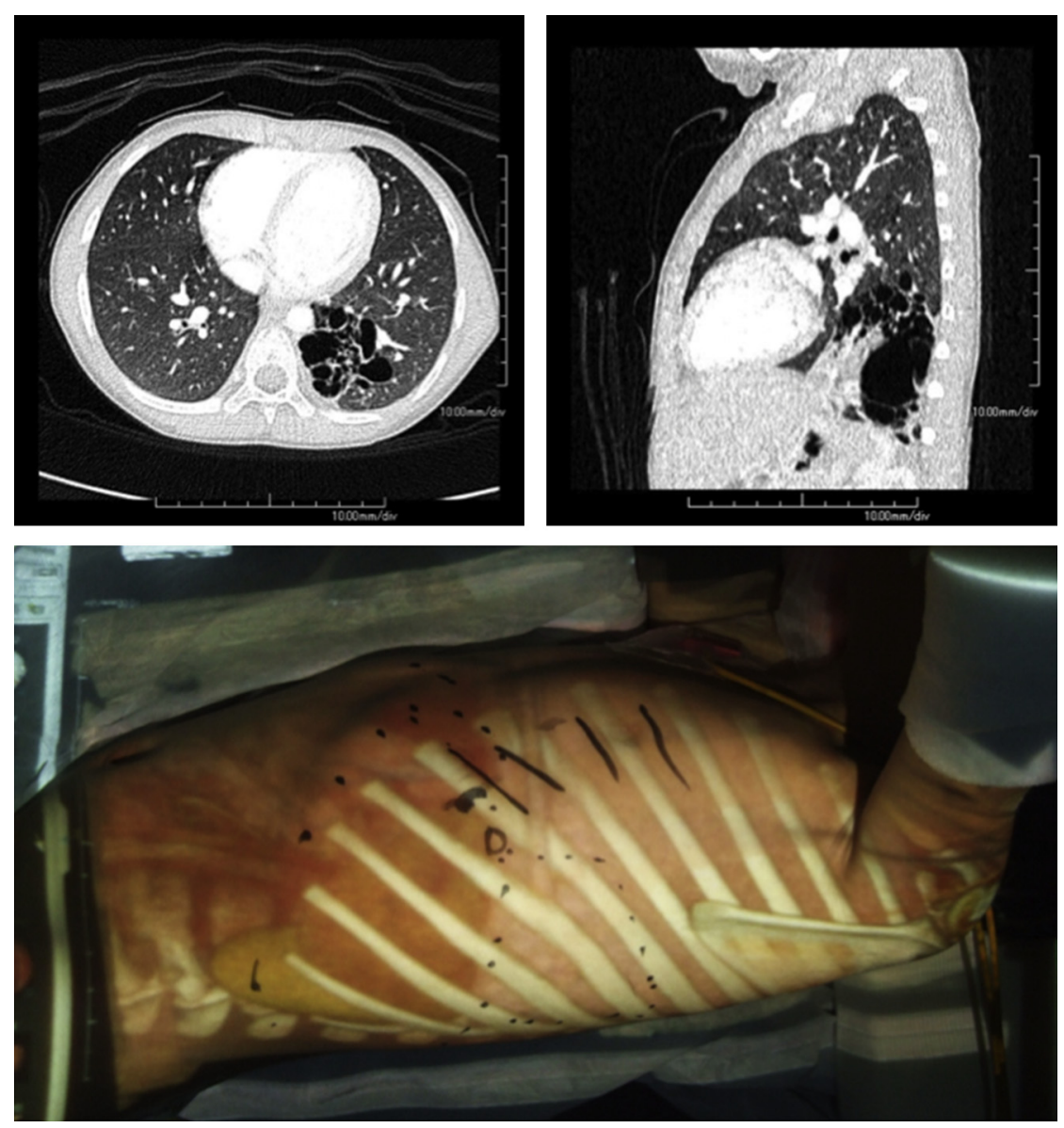

FIGURE 1. The thoracic cage and affected lung were projected onto the body surface of a 5-year-old girl, facilitating the placement of trocars for the performance of a thoracoscopic anatomic left lateral and posterior basal segmentectomy. 
A commercially available liquid crystal projector was mounted directly onto the surgical light arm so that the reconstructed images could be projected directly onto the patient's skin (Figure 1). The clavicle, lower costal edge, and iliac crest were used as reference points to fit the image to the patient. The projected image allowed the surgeon to navigate through the patient's body, going from the surface, the skin, to the pulmonary hilum. Thus, the patient's anatomy could be used to guide trocar placement to avoid damage to surrounding structures.

\section{PRACTICAL APPLICATION}

At the present stage of development, the projector has to be put away after imaging; therefore, we apply the method only for cases in which trocar placement is considered to be problematic. Since November 2010, we have used this method for 1 pediatric lobectomy, 1 pediatric segmentectomy, 1 pleural biopsy, 2 localized empyema debridement or adhesiolysis procedures, and 8 lung segmentectomies. In cases of empyema, the cavity can usually be detected using ultrasonography. However, our 2 cases were referred because of drainage failure, because there was a residual amount of air after tube removal that made the space invisible to the ultrasonic device. Our method of projecting the space and the rib cage onto the patient was helpful in identifying the air-containing space.

Thoracoscopic lateral or posterior basal segmentectomy is difficult because of its complex anatomy. ${ }^{1}$ Projecting vascular information directly onto the patient allowed the surgeon to determine the position and direction of the vessels. Furthermore, the selection of an appropriate port position in relation to the intersegmental plane that was to be dissected helped preclude complicated maneuvers. For segmentectomies classified as difficult, according to a previous report, ${ }^{1}$ the mean operation time was reduced from 254 to 184 minutes with the application of the new method.

\section{DISCUSSION}

The current routine method for thoracic port insertion depends on surgeons' experience or intuition, and even an experienced surgeon might be perplexed in some cases. The 3D projection method proposed herein offers several advantages similar to those reported in neurosurgery and other fields. ${ }^{2-4}$ First, each 3D object can be made translucent, removed, or rotated with a mouse-controlled cursor, according to the patient's position. Second, the method is noninvasive. Third, although an experienced surgeon may be able to select appropriate port positions automatically, an appreciation of the patient's real anatomy will facilitate this process.

The method could also be of educational value. Although a surgeon is familiar with the morphology of thoracic structures, visualization of the associated anatomy via a $3 \mathrm{D}$ image could assist young surgeons or students in understanding spatial tissue relationships and visualizing various approaches preoperatively.

The image overlay technique can help avoid inappropriate instrument placement, thereby reducing risks and perioperative complications. Before the introduction of this method, we encountered 2 cases of diaphragmatic injury with port placement in patients with localized pleural disease, whereas there has been no such complication with the new method. This method also allows the determination of the port position in relation to the pulmonary arteries and veins to manage the dissection of intersegmental lung parenchyma. ${ }^{1,5}$ In particular, it has been useful for predicting the appropriate port position for segmentectomy of the posterior and lateral basal segments, a task considered difficult during a thoracoscopic procedure. ${ }^{1}$ Thus, our experience suggests that the image overlay technique can be of material assistance in trocar placement and is useful in planning the therapeutic approach for patients with various conditions.

Although there is some scope for improving the image accuracy and device setting method, we believe that this technique could be a valuable aid in achieving optimum trocar placement for thoracoscopic surgery.

\section{References}

1. Oizumi H, Kanauchi N, Kato H, Endoh M, Suzuki J, Fukaya K, et al. Anatomic thoracoscopic pulmonary segmentectomy under 3-dimensional multidetector computed tomography simulation: a report of 52 consecutive cases. $J$ Thorac Cardiovasc Surg. 2011;141:678-82.

2. Nakajima S, Atsumi H, Kikinis R, Moriarty TM, Metcalf DC, Jolesz FA, et al. Use of cortical surface vessel registration for image-guided neurosurgery. Neurosurgery. 1997;40:1201-8.

3. Blackwell M, Nikou C, DiGioia AM, Kanade T. An image overlay system for medical data visualization. Med Image Anal. 2000;4:67-72

4. Tagaya N, Aoyagi H, Nakagawa A, Abe A, Iwasaki Y, Tachibana M, et al. A novel approach for sentinel lymph node identification using fluorescence imaging and image overlay navigation surgery in patients with breast cancer. World J Surg. 2011;35:154-8

5. Oizumi H, Endoh M, Takeda S, Suzuki J, Fukaya K, Sadahiro M. Anatomical lung segmentectomy simulated by computed tomographic angiography. Ann Thorac Surg. 2010;90:1382-3 